\title{
High adherence to the Western, Prudent, and Mediterranean dietary patterns and risk of gastric adenocarcinoma: MCC-Spain study
}

\author{
Adela Castelló ${ }^{1,2,3}$ - Nerea Fernández de Larrea ${ }^{1,2} \cdot$ Vicente Martín $^{4} \cdot$ Verónica Dávila-Batista ${ }^{4}$. \\ Elena Boldo ${ }^{1,2}$. Marcela Guevara ${ }^{2,5}$. Víctor Moreno ${ }^{2,6,7}$. Gemma Castaño-Vinyals ${ }^{2,8,9,10} \cdot$ Inés Gómez-Acebo ${ }^{11}$. \\ Guillermo Fernández-Tardón ${ }^{12} \cdot$ Rosana Peiró ${ }^{2,13} \cdot$ Rocío Olmedo-Requena $^{2,14,15} \cdot$ Rocio Capelo $^{16}$. \\ Carmen Navarro, ${ }^{2,17,18}$. Silvino Pacho-Valbuena ${ }^{19}$. Beatriz Pérez-Gómez ${ }^{1,2}$ - Manolis Kogevinas ${ }^{2,8,9,10}$. \\ Marina Pollán ${ }^{1,2} \cdot$ Nuria Aragonés ${ }^{1,2} \cdot$ On behalf of the MCC-Spain researchers
}

Received: 13 July 2017 / Accepted: 10 October 2017 / Published online: 14 November 2017

(c) The International Gastric Cancer Association and The Japanese Gastric Cancer Association 2017

\begin{abstract}
Background The influence of dietary habits on the development of gastric adenocarcinoma is not clear. The objective of the present study was to explore the association of three previously identified dietary patterns with gastric adenocarcinoma by sex, age, cancer site, and morphology.

Methods MCC-Spain is a multicase-control study that included 295 incident cases of gastric adenocarcinoma and 3040 controls. The association of the Western, Prudent, and Mediterranean dietary patterns-derived in another Spanish casecontrol study - with gastric adenocarcinoma was assessed using multivariable logistic regression models with random province-specific intercepts and considering a possible interaction with sex and age. Risk according to tumor site (cardia, non-cardia) and morphology (intestinal/diffuse) was evaluated using multinomial regression models.

Results A high adherence to the Western pattern increased gastric adenocarcinoma risk [odds ratio $_{\text {fourth_vs._first_quartile }}(95 \%$

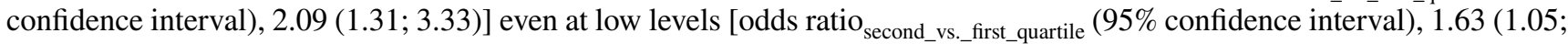
2.52)]. High adherence to the Mediterranean dietary pattern could prevent gastric adenocarcinoma [odds ratio fourth_vs._first_quartile $_{\text {. }}$ (95\% confidence interval), $0.53(0.34 ; 0.82)]$. Although no significant heterogeneity of effects was observed, the harmful effect of the Western pattern was stronger among older participants and for non-cardia adenocarcinomas, whereas the protective effect of the Mediterranean pattern was only observed among younger participants and for non-cardia tumors.

Conclusion Decreasing the consumption of fatty and sugary products and of red and processed meat in favor of an increase in the intake of fruits, vegetables, legumes, olive oil, nuts, and fish might prevent gastric adenocarcinoma.
\end{abstract}

Keywords Diet, Mediterranean $\cdot$ Diet, Western $\cdot$ Stomach neoplasms $\cdot$ Adenocarcinoma $\cdot$ Prevention and control $\cdot$ Principal component analysis $\cdot$ Population attributable fraction

\section{Introduction}

Although age-standardized incidence rates of gastric cancer (GC) have decreased globally during the past decades, this tumor continues to be the fifth most often diagnosed worldwide, and the absolute number of new cases has increased

Electronic supplementary material The online version of this article (doi:10.1007/s10120-017-0774-x) contains supplementary material, which is available to authorized users.

Adela Castelló

acastello@isciii.es

Extended author information available on the last page of the article from nearly 1.2 million in 2005 to more than 1.3 million in 2015 [1]. Also, because of its poor survival rates, GC ranks third in mortality worldwide [1]. Therefore, in the following years, cancer prevention efforts should be as important as related delivery of care.

Even though the global burden of GC that is attributable to Helicobacter pylori ( $H$. pylori) is estimated to be around $89 \%$ [2], some authors suggest that diet might also have an important role [3-6]. However, the last report on updated evidence on Food, Nutrition, Physical Activity, and the Prevention of GC published in 2016 by the World Cancer Research Fund and the American Institute of Cancer Research (WCRF/AICR) concludes that there is only strong 
evidence for a detrimental effect of a high consumption of alcohol, salt-preserved foods, and processed meat in GC risk and suggests a possible protective effect of citrus fruits for some types of GC tumors [5].

In the past decades some authors have argued that the lack of conclusive associations between diet and some diseases might be because the effects of foods and nutrients are usually explored individually [7-9], and they suggest that dietary pattern analysis would be more adequate because it allows exploration of the effect of food and nutrient interactions in disease [7-9]. Although two recent reviews [10,11] confirm the potential preventive effect of a diet labeled as "Prudent/Healthy" and the detrimental effect of the so-called "Western/Unhealthy," the WCRF/AICR does not consider the evidence sufficient to include the effect of these diets as conclusively related to GC [5].

A recent Spanish study on female breast cancer (BC) (EpiGEICAM) identified three data-driven dietary patterns [12]: a Western pattern associated with increased risk, a Prudent pattern not associated with BC, and a protective Mediterranean pattern. The EpiGEICAM study presents the novelty of being able to identify, with data-driven statistical methods and over a single population, two patterns that are commonly interchanged in the related literature (Prudent and Mediterranean). According to this study, these two patterns represent two diets with different characteristics that might be determinant in their association with disease risk [12]. We believe that the application of these patterns in different populations and the exploration of their association with tumors other than BC are of great scientific interest. In fact, these patterns have already been applied over an independent sample, and the reproducibility of the results obtained in EpiGEICAM has been assessed for breast [13] and prostate cancer [14].

The objective of the present study is to assess the reproducibility of the associations found between a high adherence to the Western, Prudent, and Mediterranean dietary patterns and $\mathrm{BC}$ risk in our country, with gastric adenocarcinoma (GAC) by sex, age, cancer site, and morphology.

\section{Methods}

The multicase-control study MCC-Spain [15] was conducted with the objective of identifying environmental, demographic, anthropometric, lifestyle, and genetic factors related to five common cancers: breast, prostate, colorectal, gastric, and chronic lymphocytic leukemia. Cases were recruited in 23 hospitals from 12 Spanish provinces (Asturias, Barcelona, Cantabria, Gerona, Granada, Guipúzcoa, Huelva, León, Madrid, Murcia, Navarra, and Valencia). A single set of population-based controls, frequency matched by age and sex with the overall distribution of cases in each province, was randomly selected from the list of residents assigned to selected primary care health centers located within the catchment area of each of the collaborating hospitals. Controls were contacted by phone, and those who agreed to participate attended a personal interview. For the specific case of GC, MCC-Spain recruited 459 histologically confirmed cases and 3440 population controls between September 2008 and December 2013 in 10 of the 12 participating provinces (all except Gerona and Guipúzcoa). The detailed selection process of GC cases and controls has been previously described elsewhere [15, 16]. Briefly, participants able to answer the questionnaire, who had lived in the study area for at least 6 months before the diagnosis, and were 20-85 years old were invited to participate. Cases were identified, as soon as possible after their diagnosis, through active search that included periodic visits to the collaborating hospital departments. Histologically confirmed incident cases of GC (codes C16: malignant neoplasm of stomach; D00.2: carcinoma in situ of stomach; and C15.5: malignant neoplasm of lower third of esophagus; of the tenth revision of the International Statistical Classification of Diseases ICD-10) with no prior history of the disease, and diagnosed within the recruitment period, were included. Tumors were classified according to their location in cardia and non-cardia and by tumor morphology following Lauren's classification into intestinal or diffuse $[17,18]$. Classification into cardia and non-cardia gastric cancer cases was done according to the information available in the medical records. Cardia cases included tumors described as located in the "esophagogastric junction" or in the "cardia." Non-cardia cases included those located distal to the esophagogastric junction (fundus, body, antrum, and pylorus). Controls were randomly selected from general practitioner lists in the same areas. The response rate was $57 \%$ among cases and $53 \%$ among controls.

The protocol of MCC-Spain was approved by each of the Ethics Committees of the participating institutions. The specific study reported here was approved by the Instituto de Salud Carlos III Ethics Committee. All participants were informed about the study objectives and signed an informed consent.

A structured computerized epidemiological questionnaire was administered by trained personnel in a face-to-face interview to collect information on sociodemographic factors, lifestyle, and personal/family medical history, among other factors. Missing values on key variables and specific questions on additional study objectives were completed through subsequent telephone contact. Height and weight at different ages were self-reported, and diet was assessed with a 154-item semi-quantitative food frequency questionnaire (FFQ), which was based on a validated instrument in Spain [19] modified to include regional products. Dietary 
information referred to the previous year before diagnosis in cases and before interview in controls.

All participants were asked for donation of blood samples, which were processed, aliquoted, and stored at $-80{ }^{\circ} \mathrm{C}$ in the first $48 \mathrm{~h}$. Only 235 (61\%) of cases and 1900 (64\%) controls donated a blood sample. From these participants, an aliquot of serum was sent on dry ice to the German Cancer Research Centre (DKFZ), Heidelberg, Germany, for the $H$. pylori multiplex serology assay [20].

In the present work, three dietary patterns identified in a previous Spanish case-control study (EpiGEICAM) that explored the association between dietary patterns and female BC risk [12] are examined: a Western dietary pattern positively associated with $\mathrm{BC}$ risk that is characterized by high intakes of high-fat dairy products, processed meat, refined grains, sweets, caloric drinks, convenience food, and sauces and by low intakes of low-fat dairy products and whole grains; a Prudent pattern with no relationship with $\mathrm{BC}$ that represented high intakes of low-fat dairy products, vegetables, fruits, whole grains, and juices; and a Mediterranean pattern that seemed to be protective and denoted a high intake of fish, vegetables, legumes, boiled potatoes, fruits, olives, and vegetable oil, represented by $72 \%$ of olive oil consumption, $23 \%$ of olives intake, and the remaining $5 \%$ coming from sunflower, corn, or soybean oil consumption among controls, and a low intake of juices. The three dietary patterns were identified in the EpiGEICAM study by grouping all the items of the FFQ used into 26 intercorrelated food groups. Subsequently, principal component analysis without rotation of the variance-covariance matrix was applied over these 26 food groups [21], obtaining a set of weights (pattern loadings in Table 1) that represents the correlation between food consumption and the component/ pattern scores and can be used to reproduce such patterns in other samples, as explained in detail elsewhere [22, 23]. To apply these patterns to the MCC-Spain sample, we grouped 146 of the 154 items of the FFQ (excluding non-caloric and alcoholic beverages) into the same 26 food groups described in EpiGEICAM (Table 1) and calculated the scores for the level of adherence to the Western, Prudent, and Mediterranean dietary patterns of the MCC-Spain participants as a linear combination of the weights of each food group and pattern published in the EpiGEICAM study (Table 1) [12] and consumption reported by the MCC-Spain participants in the current study for each food group.

After describing the sample with basic descriptive statistics, adjusted associations between adherence to each dietary pattern and GAC risk were evaluated using logistic regression models with random province-specific intercepts. As fixed-effects terms, sex, age, education, body mass index (BMI), family history of gastric cancer, physical activity [metabolic equivalents (METs)] during the 10 years before diagnosis/interview, smoking status, $H$. pylori seropositivity, and caloric and alcohol intake were considered as potential confounders. Scores of adherence were analyzed both as categorical (grouping the scores of adherence into quartiles of their distribution among controls) and continuous (1-standard deviation increase taking into account the dispersion among controls) variables.

Because including data on $\mathrm{H}$. pylori seropositivity implied losing a large part of the sample (36\% of the participants with complete data on the variables included in the analyses), an initial sensitivity analysis was carried out to choose the best modeling strategy (see Table S1 from Online Resource 1). The results from five models were compared. Model 1 included only the province of residence as a random effect; Model 2 was adjusted for all the potential confounders except $H$. pylori seropositivity; to estimate the effect of reducing the sample size when including $H$. pylori data independently from its confounding effect; Model 3 included the same set of potential confounders as Model 2, but analyses were restricted to those individuals with information on $H$. pylori infection. Model 4 was adjusted by all potential confounders, including $H$. pylori infection status, to assess the possible confounding effect of this variable; and Model 5 included the same set of potential confounders as Model 2 but was restricted to $H$. pylori-positive participants (89\% of those with $H$. pylori data) to check the associations in this specific group. The direction of the associations found was similar for all the analyses, and the conclusions of the study did not differ among modeling strategies except for the power of the study to detect statistically significant estimations. Differences found between models with and without $H$. pylori information were caused by a loss of power when including in the models only individuals with blood samples and not by a confounding effect of this variable, as is shown by the different results found for Models 2 and 3 in contrast with the very similar results obtained from Models 3 and 4. Taking this into account, and to keep the maximum statistical power for further estimations, we decided to select the modeling strategy from Model 2 for all the analyses included in Tables 3 and 4.

Heterogeneity of the effects of each dietary pattern by sex and age group (defined by the median age among cases to ensure equal distribution of individuals among groups: $\leq 68$ and $>68$ years old) was tested, including in the models an interaction term between these two variables and the score of adherence to each of the three dietary patterns under study.

Multinomial logistic regression models were used to evaluate the association of the adherence to the Western, Prudent, and Mediterranean dietary patterns with GAC by location (cardia and non-cardia) and morphology (intestinal and diffuse). All these models were adjusted for sex, age, education, BMI, family history of gastric cancer, and physical activity (METs) during the 10 years before diagnosis/ 
Table 1 Composition of food groups based on the food frequency questionnaire of the MCC-Spain study and component loadings for each pattern identified in the previous study [12]

\begin{tabular}{|c|c|c|c|c|}
\hline Food group & Food $^{\mathrm{a}}$ & $W$ & $P$ & $M$ \\
\hline High-fat dairy & $\begin{array}{l}\text { Whole-fat milk, condensed milk, whole-fat yogurt, semi-cured, cured, or creamy cheese, } \\
\text { blue cheese, custard, milkshake, ice cream, double cream }\end{array}$ & 0.60 & -0.11 & 0.20 \\
\hline Low-fat dairy & $\begin{array}{l}\text { Semi-skimmed and skimmed milk, soy milk, skimmed yogurt, curd, cottage or fresh } \\
\text { white cheese }\end{array}$ & -0.49 & 0.60 & -0.01 \\
\hline Eggs & Eggs & 0.19 & 0.08 & 0.16 \\
\hline White meat & Chicken, rabbit, duck & 0.08 & 0.17 & 0.18 \\
\hline Red meat & $\begin{array}{l}\text { Pork, beef, lamb, liver (beef, pork or chicken), entrails, hamburgers (pork or beef) and } \\
\text { meatballs (pork or beef) }\end{array}$ & 0.27 & 0.09 & 0.22 \\
\hline Processed meat & Sausages, Serrano ham and other cold meat, bacon, pâté, foie gras & 0.36 & 0.10 & 0.26 \\
\hline White fish & Fresh or frozen white fish (hake, sea bass, sea bream), $1 / 2$ salted fish and $1 / 2$ smoked fish & 0.01 & 0.24 & 0.34 \\
\hline Oily fish & $\begin{array}{l}\text { Fresh or frozen bluefish (tuna, swordfish, sardines, anchovies, salmon), canned fish, } 1 / 2 \\
\text { salted fish and } 1 / 2 \text { smoked fish }\end{array}$ & 0.05 & 0.24 & 0.44 \\
\hline Seafood/shellfish & Clams, mussels, oysters, squid, cuttlefish, octopus, prawn, crab, shrimp, similar products & 0.17 & 0.27 & 0.35 \\
\hline Leafy vegetables & Spinach, chard, lettuce, other leafy vegetables & -0.11 & 0.34 & 0.40 \\
\hline Fruiting vegetables & Tomato, eggplant, zucchini, cucumber, pepper, artichoke, avocado & 0.00 & 0.36 & 0.45 \\
\hline Root vegetables & Carrot, pumpkin, radish & 0.05 & 0.35 & 0.44 \\
\hline Other vegetables & $\begin{array}{l}\text { Cooked cabbage, cauliflower, or broccoli, onion, green beans, asparagus, mushrooms, } \\
\text { corn, garlic, gazpacho, vegetable soup, other vegetables }\end{array}$ & -0.04 & 0.40 & 0.42 \\
\hline Legumes & Peas, lentils, chickpeas, beans, broad beans & 0.21 & 0.15 & 0.34 \\
\hline Potatoes & Roasted or boiled potatoes and sweet potatoes & 0.17 & 0.25 & 0.40 \\
\hline Fruits & $\begin{array}{l}\text { Orange, grapefruit, mandarin, banana, apple, pear, grapes, kiwi, strawberries, cherries, } \\
\text { peach, figs, melon or watermelon, prunes, mango, papaya, other fresh or dried fruits }\end{array}$ & -0.07 & 0.31 & 0.31 \\
\hline Nuts & Almonds, peanuts, pine nuts, hazelnut & 0.18 & 0.22 & 0.29 \\
\hline Refined grains & White flour bread, rice, pasta & 0.37 & 0.15 & 0.23 \\
\hline Whole grains & Whole-grain bread and breakfast cereals & -0.43 & 0.47 & -0.06 \\
\hline Olives and vegetable oil & $\begin{array}{l}\text { Olives, added olive oil to salads, bread, and dishes, other vegetable oils (sunflower, corn, } \\
\text { soybean) }\end{array}$ & 0.12 & 0.19 & 0.34 \\
\hline Other edible fats & Margarine, butter, lard & 0.22 & 0.02 & 0.11 \\
\hline Sweets & $\begin{array}{l}\text { Chocolate and other sweets, cocoa powder, plain cookies, chocolate cookies, pastries } \\
\text { (croissant, donut, cake, pie, etc.) }\end{array}$ & 0.35 & 0.18 & 0.05 \\
\hline Sugary & Jam, honey, sugar, and fruit in sugar syrup & 0.24 & 0.05 & 0.00 \\
\hline Juices & Tomato juice, freshly squeezed orange juice, juice (other than freshly squeezed) & 0.25 & 0.67 & -0.39 \\
\hline Caloric drinks & Sugar-sweetened soft drinks and nut milk & 0.74 & 0.21 & -0.25 \\
\hline Convenience food and sauces & $\begin{array}{l}\text { Croquette, fish sticks, dumplings, kebab, fried potatoes, crisps, pizza, instant soup, may- } \\
\text { onnaise, tomato sauce, hot sauce, ketchup, other sauces }\end{array}$ & 0.47 & 0.12 & 0.24 \\
\hline
\end{tabular}

$W$ Western, $P$ Prudent, $M$ Mediterranean

${ }^{\text {a }}$ Log-transformed centered intake in grams

interview, smoking status, caloric and alcohol intake, and province of residence.

Finally, assuming a causal relationship between adherence to each of the patterns and GC for all analyses, the population attributable fraction (PAF\%) was calculated using Levin's formula [24], modified by Hanley [25] to accommodate exposures with more than one category. The PAF\% describes the proportion of gastric cancer in this population that hypothetically would not have occurred if all participants were in the optimal quartile of adherence to the dietary patterns (first quartile for Western and Prudent dietary patterns and fourth quartile for the Mediterranean). Confidence intervals for the PAF were computed using bootstrap with 500 iterations.

Analyses were performed using STATA/MP (version 14.1, 2015, StataCorp LP) and statistical significance was set at two-sided $p<0.05$.

\section{Results}

Initially, 3440 controls and 459 cases of GC were recruited. Among them, 3040 (88\%) controls and 354 $(77 \%)$ cases reported data on diet. Cases that provided 
dietary information later than 6 months after diagnosis were excluded $(n=40)$. Tumors other than adenocarcinomas $(n=19)$ were also excluded from the analyses. Therefore 295 GAC cases and 3040 controls aged 23-85 years were included in the present study.

Compared to controls, GAC cases showed a higher adherence to the three dietary patterns and reported higher energy and alcohol intake than controls in the univariable analyses. The proportion of males was higher among GAC cases that were also older and reported lower levels of physical activity and formal education (Table 2).

Table 3 summarizes the adjusted ORs for the association between GAC incidence and the scores of adherence to Western, Prudent, and Mediterranean dietary patterns, for the whole sample and stratified by sex and age. A higher adherence to the Western pattern was associated with higher odds of GAC even for moderate adherence, going from a $63 \%$ increased risk for participants in the second quartile of adherence [ $\left.\mathrm{OR}_{\text {second vs. first quartile }}(95 \% \mathrm{CI}), 1.63(1.05 ; 2.52)\right]$, to a more than twofold increased risk for participants in the third and fourth quartiles of adherence $\left[\mathrm{OR}_{\text {third vs. first quartile }}\right.$ (95\% CI), $2.23(1.45 ; 3.43)$, and $\mathrm{OR}_{\text {fourth vs. first quartile }}(95 \%$ CI), 2.09 (1.31; 3.33)]. Risks were very similar by sex ( $p$ interaction $=0.799)$ and age $(p$ interaction $=0.398)$ groups, but data suggest that the deleterious effect of moderate adherences to the Western dietary pattern might be stronger for older [ $>68: \mathrm{OR}_{\text {second vs. first quartile }}(95 \% \mathrm{CI}), 1.88(1.04$; $3.41)$, and $\left.\mathrm{OR}_{\text {third vs. first quartile }}(95 \% \mathrm{CI}), 2.74(1.54 ; 4.88)\right]$ than for younger $\left[\leq 68: \mathrm{OR}_{\text {second vs. first quartile }}(95 \% \mathrm{CI}), 1.39\right.$ $(0.74 ; 2.72)$, and $\mathrm{OR}_{\text {third vs. first quartile }}(95 \% \mathrm{CI}), 1.83(0.99$;
Table 2 Description of scores of adherence to Western, Prudent, and Mediterranean dietary patterns and other baseline characteristics for gastric adenocarcinoma cases and controls

\begin{tabular}{|c|c|c|c|}
\hline & $\begin{array}{l}\text { Controls } \\
n=3040\end{array}$ & $\begin{array}{l}\text { Cases } \\
n=295\end{array}$ & $p$ \\
\hline Age (years) mean (SD) & $63.93(11.43)$ & $65.89(12.63)$ & 0.005 \\
\hline $\operatorname{Sex}, n(\%)^{\mathrm{a}}$ & & & $<0.001$ \\
\hline Male & $1692(56 \%)$ & $207(70 \%)$ & \\
\hline Female & $1348(44 \%)$ & $88(30 \%)$ & \\
\hline Education, $n(\%)^{\mathrm{a}}$ & & & $<0.001$ \\
\hline No formal education & $545(18 \%)$ & $82(28 \%)$ & \\
\hline Primary school & $1027(34 \%)$ & $113(38 \%)$ & \\
\hline Secondary school & $852(28 \%)$ & $69(23 \%)$ & \\
\hline University or more & $616(20 \%)$ & $31(11 \%)$ & \\
\hline Western mean (SD) & $-0.34(3.49)$ & $0.84(3.48)$ & $<0.001$ \\
\hline Prudent mean (SD) & $-0.10(3.30)$ & $0.36(3.35)$ & 0.022 \\
\hline Mediterranean mean (SD) & $0.05(2.88)$ & $0.41(2.54)$ & 0.043 \\
\hline Energy (kcal/day) mean (SD) & $1912.50(571.94)$ & $2095.19(651.32)$ & $<0.001$ \\
\hline Alcohol (g/day) median (IQR) & $7.57(0.00 ; 24.72)$ & $12.92(1.41 ; 40.42)$ & $<0.001$ \\
\hline BMI $\left(\mathrm{kg} / \mathrm{m}^{2}\right)$, mean (SD) & $26.70(4.33)$ & $27.15(3.87)$ & 0.094 \\
\hline Physical activity & & & $<0.001$ \\
\hline 0 METs/week & $1189(39 \%)$ & $147(50 \%)$ & \\
\hline 0.1-8 METs/week & $410(13 \%)$ & $33(11 \%)$ & \\
\hline 8.1-15.9 METs/week & $355(12 \%)$ & $15(5 \%)$ & \\
\hline$\geq 16 \mathrm{METs} /$ week & $1048(34 \%)$ & $100(34 \%)$ & \\
\hline Unknown & $38(1 \%)$ & $0(0 \%)$ & \\
\hline Smoking, $n(\%)^{\mathrm{a}}$ & & & 0.703 \\
\hline Never smoker & $1332(44 \%)$ & $123(42 \%)$ & \\
\hline Former smoker & $1092(36 \%)$ & $104(35 \%)$ & \\
\hline Current smoker & $604(20 \%)$ & $67(23 \%)$ & \\
\hline Unknown & $12(0 \%)$ & $1(0 \%)$ & \\
\hline Family history of GC, $n(\%)^{\mathrm{a}}$ & & & $<0.001$ \\
\hline No & $2707(89 \%)$ & $231(78 \%)$ & \\
\hline Second degree & $139(5 \%)$ & $14(5 \%)$ & \\
\hline One of first degree & $182(6 \%)$ & $43(15 \%)$ & \\
\hline More than one of first degree & $12(0 \%)$ & $7(2 \%)$ & \\
\hline
\end{tabular}

$S D$ standard deviation, $B M I$ body mass index, $G C$ gastric cancer, METS metabolic equivalent

${ }^{\text {a }}$ Percentages might not add up 100 because of rounding 


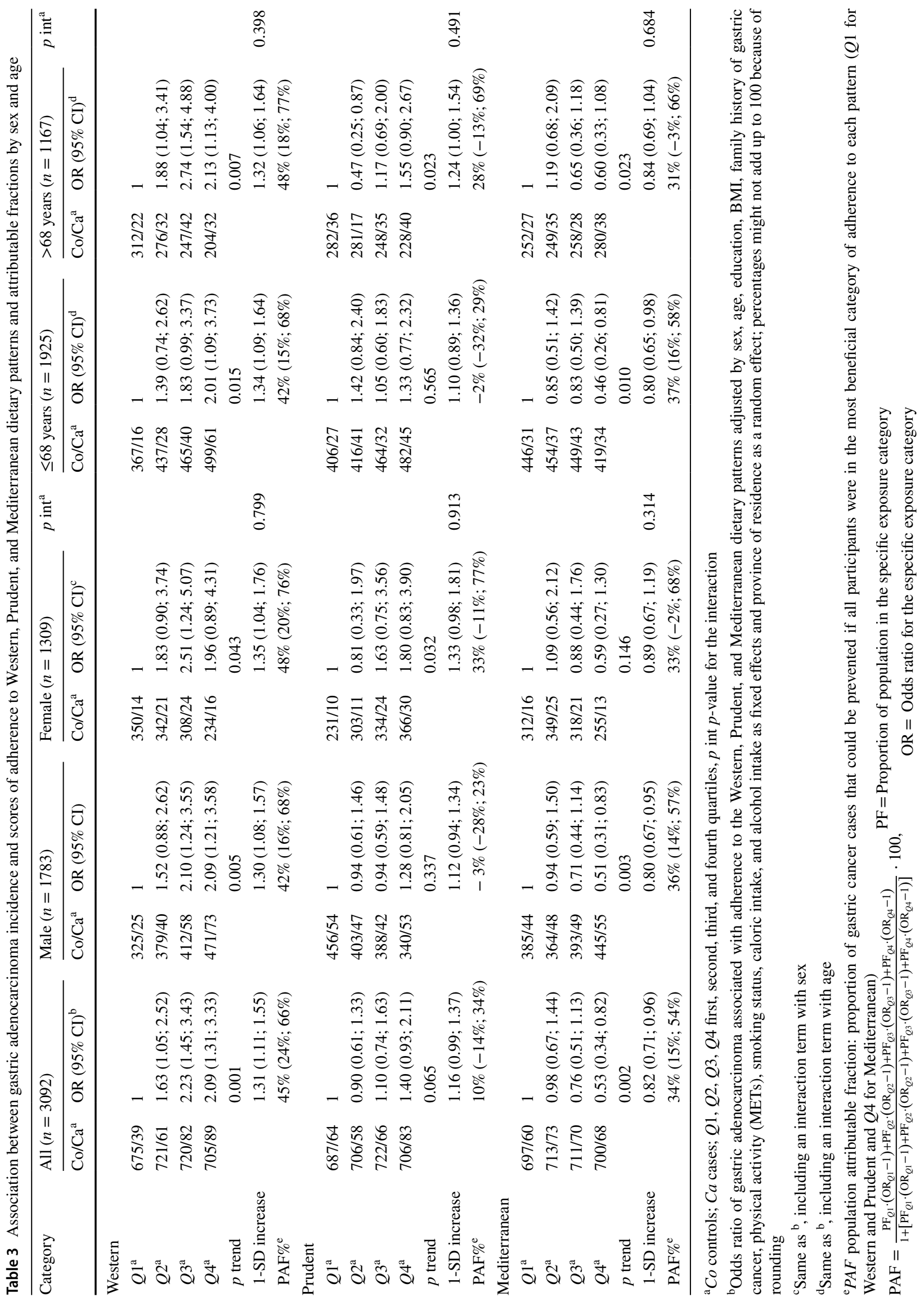


3.37)] individuals. Although no clear effect was observed between GAC and the adherence to the Prudent dietary pattern, a high adherence to the Mediterranean dietary pattern seems to have a considerable protective effect against this tumor, with a significant linear trend. Participants in the highest category of adherence to the Mediterranean dietary pattern compared to those in the lowest category had an odds ratio of developing GAC of 0.53 (95\% CI, 0.34; 0.82). Even if heterogeneity of the effects was not statistically significant $\left(p\right.$ interaction $_{\mathrm{sex}}=0.314$ and $p$ interaction $\left._{\mathrm{age}}=0.684\right)$, our data suggest that this effect might be stronger among males $\left[\mathrm{OR}_{\text {fourth vs. first quartile }}(95 \% \mathrm{CI}), 0.51(0.31 ; 0.83)\right]$ than among females $\left[\mathrm{OR}_{\text {fourth vs. first quartile }}(95 \% \mathrm{CI}), 0.59(0.27 ; 1.30)\right]$ and among younger [ $\left[\mathrm{OR}_{\text {fourth vs. first quartile }}(95 \% \mathrm{CI}), 0.46(0.26\right.$; $0.81)]$ than among older $\left[\mathrm{OR}_{\text {fourth vs. first quartile }}(95 \% \mathrm{CI}), 0.60\right.$ $(0.33 ; 1.08)]$ participants. In concordance with these results and assuming a causal relationship between the adherence to these dietary patterns and GAC risk, the estimations indicate that $45 \%$ (95\% CI, 24\%; 66\%) of GAC cases could have been prevented if all the participants had been in the lowest category of adherence to the Western pattern and that $34 \%$ (95\% CI, $15 \%$; $54 \%)$ of GAC cases could have been prevented if all the participants had the highest adherence to the Mediterranean pattern. Such PAFs\% were very similar for males and females and for younger and older participants. The positive trend found for the Western dietary pattern and the inverse trend found for the Mediterranean dietary pattern in the overall analyses was also observed by tumor location and morphology (Table 4). However, dose-response trends were only statistically significant for non-cardia tumors, for both the Western pattern [cardia: $\mathrm{OR}_{1-\mathrm{SD} \text { increase }}(95 \% \mathrm{CI})$, 1.29 ( $0.93 ; 1.77)$; non-cardia: $\mathrm{OR}_{1 \text {-SD increase }}(95 \% \mathrm{CI}), 1.35$ $(1.12 ; 1.63) ; p$ heterogeneity $=0.800]$ and the Mediterranean pattern [cardia: $\mathrm{OR}_{1-\mathrm{SD} \text { increase }}(95 \% \mathrm{CI}), 0.91(0.67 ; 1.24)$; non-cardia: $\mathrm{OR}_{1-\mathrm{SD} \text { increase }}(95 \% \mathrm{CI}), 0.78$ (0.66; 0.93); $p$ heterogeneity $=0.395]$. No clear differences were observed regarding tumor morphology. The associations between Western and Mediterranean patterns were very similar in both intestinal and diffuse tumors.

\section{Discussion}

The associations observed for BC in EpiGEICAM were also found for GAC in the MCC-Spain study. Our results suggest that a high adherence to the Western dietary pattern might increase the risk of developing GAC even for low adherence, and that high adherence to the Mediterranean dietary pattern could prevent GAC. It is also highlighted that the Prudent pattern, in spite of sharing some characteristics with the Mediterranean diet, has no clear effect on GAC risk. Our data also point out to a possible stronger effect of the Western pattern among older participants and for non-cardia tumors and a larger influence of the Mediterranean pattern on males, younger participants, and for non-cardia tumors, even though tests of heterogeneity were not statistically significant.

Most of the studies exploring the association between data-driven dietary patterns and GC risk identify two types of dietary patterns: a Western/Unhealthy pattern and a Healthy/Prudent pattern. The first one usually loads high in red and processed meat, sweets, soft drinks, high-fat dairy, fast food, and sauces and is positively associated with GC risk [26-28]. Some studies collect these foods in two different patterns (Western and Traditional/Mixed) with at least one of them positively associated with GC [29-32] and others report a null effect of the Western pattern on GC risk [33, 34]. The Mediterranean/Healthy pattern usually loads high in fruits and vegetables, fish, legumes, and vegetable oils and appears to be protective in most cases [26, 27, 29-32], with few exceptions for studies including only tumors in the cardia [28] or patterns that only contain fruits and vegetables [33], in concordance with the absence of an association we found for the Prudent pattern. Only three of these studies explored differences by tumor location [26, 27] or morphology [26, 31]. Although some authors show similar strength of the associations for cardia and non-cardia tumors [26], others claim a stronger effect of the Western and Mediterranean/Healthy dietary patterns among non-cardia tumors in females and among cardia tumors in males [27]. Unfortunately, the sample size of the present study did not allow the exploration of a possible interaction between dietary patterns and sex by tumor location. Regarding tumor morphology, although Bastos et al. [26] state that the effect of the Western pattern is only observed among intestinal adenocarcinomas, Kim et al. [31] found a stronger effect of the healthy pattern for female diffuse adenocarcinomas. However, none of the authors provide assessment of the statistical significance of the differences declared. In our case, we believe that the greater sample size of the non-cardia and intestinal tumor subtypes might be related to the greater significance of the associations found for these subgroups.

Some biological mechanisms support the plausibility of the associations found. The Western pattern includes a high consumption of red and processed meat, which contributes to the generation of $N$-nitroso compounds that are suspected mutagens and carcinogens [35]. Additionally, cooking and processing meat at high temperatures might also contribute to the production of polycyclic aromatic hydrocarbons, which are well-known carcinogens [36]. The iron present in these types of meat might also induce free radicals, which cause DNA double-strand breaks and oncogene activation [37]. In addition, the saturated fats that can be found in fast food, sauces, and desserts, also included in the Western diet, can induce expression of certain inflammatory mediators associated with 


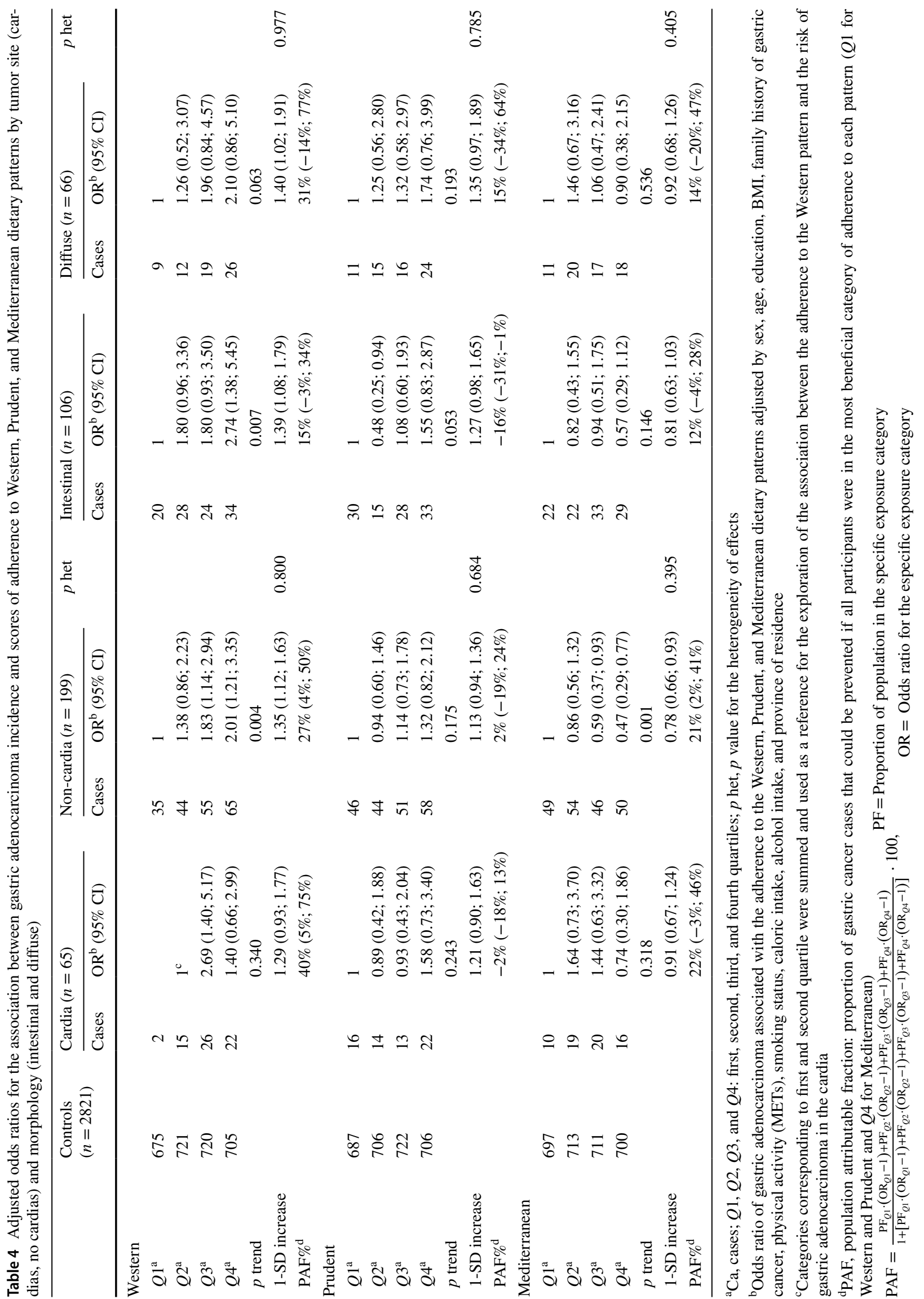


carcinogenesis [38]. Regarding the Mediterranean pattern, the high content of antioxidants in fruits and vegetables might be a partial cause of the protective effect of the Mediterranean diet. Antioxidants quench free radicals, reduce oxidative damage to DNA, and intervene in various cancer-related biological pathways such as carcinogen bio-activation, cell signaling, cell-cycle regulation, angiogenesis, and inflammation [39, 40]. Additionally, Allium vegetables, particularly garlic, can reduce the severity of $H$. pylori-associated gastritis, and bioactive constituents in fruit might protect against $H$. pylori-induced damage, particularly inflammation, which is implicated in the development of gastric cancers [41]. Moreover, omega-3 polyunsaturated fatty acids, present in fish and nuts, may have preventive effects by influencing multiple targets implicated in various stages of cancer development, including cell proliferation, cell survival, angiogenesis, inflammation, and metastasis [42].

Our results should be interpreted in the context of the study's limitations. Recall bias is always a concern in case-control studies, especially when evaluating the effect of self-reported dietary information. Anticipating the existence of this bias, some questions about general dietary habits were included in the questionnaire and used to adjust the responses to the FFQ [43]. To minimize even more the effect of this possible bias, only cases that responded to the questionnaire within the 6 months following the diagnosis were included. On the other hand, the response rate was 57\% for cases and 53\% for controls, which may appear to be low and might raise some concerns about selection bias. Participating controls might have better lifestyles, resulting in an overestimation of the effects. However, no effect was found for the prudent pattern that includes consumption of products widely known as "Healthy". We believe it is unlikely that the effect of this bias is selective and affects only some associations. Furthermore, although the literature does not reflect agreement on a minimum acceptable response rate, there is general consensus that a $50 \%$ response rate might be adequate [44]. In addition, the strength of the associations found, their consistency across sex, age, and subtypes, their consistency with the results from EpiGEICAM [12] [the Western pattern increased the risk of BC in EpiGEICAM and GAC in MCC-Spain, the Prudent pattern had no effect on these two tumors, and the Mediterranean pattern appeared to be protective against both BC (EpiGEICAM) and GAC (MCC-Spain)] and from other GC studies, as well as their biological plausibility, deem it unlikely that our findings are a result of recall or selection bias. Finally, results were not adjusted by data on $H$. pylori infection, the main explanatory cause of non-cardia GC. However, the sensitivity analyses carried out (Table S1 from Online Resource 1) showed no important differences in the estimation of the effects when taking this factor into account, thus supporting the reported associations between diet and GAC as being independent of $H$. pylori infection.

One of the strengths of the current research is the recruitment of histologically confirmed incident cases of GC and population-based controls. Additionally, the reproducibility [23] and applicability [22] of the data-driven dietary patterns found in the EpiGEICAM study [12] were methodologically tested in two recent studies [22, 23]. These studies concluded that similar patterns can be found in independent samples [23] and that scores of adherence to data-driven dietary patterns can be calculated following the exact same rules over different populations, resulting in different levels of adherence but still being valid [22]. Furthermore, the sample size allowed the evaluation of potential interactions of diet with sex and age and the exploration of the associations by tumor location and morphology. Finally, the inclusion of cases and controls recruited from ten provinces from the north, south, center, west, and east of the country ensured the representation of the different diets coexisting within Spain. The dietary variability of the participants allowed the differentiation of the effect of two very similar patterns on GAC risk. Prudent and Mediterranean dietary patterns are commonly interchanged in the literature of data-driven dietary patterns, but they showed different effects on GAC in our study, adding novel information about the association of the so-called healthy dietary habits and GAC. In addition, if our results showing a lack of protective effect of the Prudent pattern on GAC risk are confirmed, the sizes of effects estimated for the Mediterranean pattern in studies that have not differentiated it from the Prudent pattern could be underestimations of the true protective role of the Mediterranean diet.

\section{Conclusion}

A high consumption of fruits, vegetables, and whole grains together with a restriction of dietary fat is not enough to prevent GAC. The risk of this tumor might be reduced in the general population by providing dietary recommendations based on decreased consumption of high-fat dairy products, red and processed meat, refined grains, sweets, caloric drinks, convenience foods, and sauces in favor of an increase in the intake of fruits, vegetables, legumes, olive oil, nuts, and fish.

Funding The study was supported by the "Acción Transversal del Cáncer," approved by the Spanish Ministry Council on 11 October 2007, by the Consortium for Biomedical Research in Epidemiology and Public Health (CIBERESP), by the Instituto de Salud Carlos III grants, co-funded by FEDER funds: a way to build Europe-PI08/1770 (to M. Kogevinas), PI09/0773 (to J. Llorca), PI09/1286 (to V. Martín), PI09/1903 (to R. Peiró), PI09/2078 (to F.J. Caballero), PI09/1662 (to J.J. Jiménez-Moleón), PI11/01403 (to N. Aragonés), and PI12/00150 (to B. Pérez-Gómez), by the Fundación Marqués de Valdecilla grant API 10/09 (to J. Llorca), by Catalan Government DURSI grant 
2014SGR647 (to V. Moreno) and 2014SGR756 (to S. de Sanjose), by the Junta de Castilla y León Grant LE22A10-2 (to V. Martín), by the Consejería de Salud of the Junta de Andalucía Grant 2009-S0143 (to J. Alguacil), by the Conselleria de Sanitat of the Generalitat Valenciana grant AP061/10 (to R. Peiró), by the Regional Government of the Basque Country, by the Consejería de Sanidad de la Región de Murcia, by the Fundación Caja de Ahorros de Asturias, by the University of Oviedo, by the Spanish Association Against Cancer (AECC) Scientific Foundation, and by the Spanish Ministry of Economy and Competitiveness Juan de la Cierva de Incorporación Grant IJCI-201420900 (to A. Castelló). None of the sponsors intervened in any stage of the research.

\section{Compliance with ethical standards}

Conflict of interest The authors do not have conflicts of interest in connection with this paper.

Ethical statement All procedures followed were in accordance with the ethical standards of the responsible committee on human experimentation (institutional and national) and with the Helsinki Declaration of 1964 and later versions. Informed consent or substitute for it was obtained from all patients for being included in the study.

\section{References}

1. Global Burden of Disease Cancer Collaboration, Fitzmaurice C, Allen C, Barber RM, Barregard L, Bhutta ZA, et al. Global, regional, and national cancer incidence, mortality, years of life lost, years lived with disability, and disability-adjusted life-years for 32 cancer groups, 1990 to 2015: a systematic analysis for the global burden of disease study. JAMA Oncol. 2016;. doi:10.1001/ jamaoncol.2016.5688.

2. Plummer M, Franceschi S, Vignat J, Forman D, de Martel C. Global burden of gastric cancer attributable to Helicobacter pylori. Int J Cancer. 2015;136:487-90. doi:10.1002/ijc.28999.

3. de Martel C, Forman D, Plummer M. Gastric cancer: epidemiology and risk factors. Gastroenterol Clin N Am. 2013;42:219-40. doi:10.1016/j.gtc.2013.01.003.

4. Karimi P, Islami F, Anandasabapathy S, Freedman ND, Kamangar F. Gastric cancer: descriptive epidemiology, risk factors, screening, and prevention. Cancer Epidemiol Prev Biomark. 2014;23:700-13. doi:10.1158/1055-9965.EPI-13-1057.

5. WCRF/AICR. World Cancer Research Fund International/American Institute for Cancer Research. Continuous Update Project Report: Diet, Nutrition, Physical Activity and Stomach Cancer. 2016. http://www.wcrf.org/stomach-cancer-2016.

6. Whiteman DC, Wilson LF. The fractions of cancer attributable to modifiable factors: a global review. Cancer Epidemiol. 2016;. doi:10.1016/j.canep.2016.06.013.

7. Barkoukis H. Importance of understanding food consumption patterns. J Am Diet Assoc. 2007;107:234-6.

8. Hu FB. Dietary pattern analysis: a new direction in nutritional epidemiology. Curr Opin Lipidol. 2002;13:3-9.

9. Jacques PF, Tucker KL. Are dietary patterns useful for understanding the role of diet in chronic disease? Am J Clin Nutr. 2001;73:1-2.

10. Bertuccio P, Rosato V, Andreano A, Ferraroni M, Decarli A, Edefonti V, et al. Dietary patterns and gastric cancer risk: a systematic review and meta-analysis. Ann Oncol. 2013;24:1450-8. doi:10.1093/annonc/mdt108.
11. Shu L, Wang XQ, Wang SF, Wang S, Mu M, Zhao Y, et al. Dietary patterns and stomach cancer: a meta-analysis. Nutr Cancer. 2013;65:1105-15. doi:10.1080/01635581.2013.828086.

12. Castello A, Pollan M, Buijsse B, Ruiz A, Casas AM, BaenaCanada JM, et al. Spanish Mediterranean diet and other dietary patterns and breast cancer risk: case-control EpiGEICAM study. Br J Cancer. 2014;111:9. doi:10.1038/bjc.2014.434.

13. Castello A, Boldo E, Perez-Gomez B, Lope V, Altzibar JM, Martin $\mathrm{V}$, et al. Adherence to the Western, Prudent and Mediterranean dietary patterns and breast cancer risk: MCC-Spain study. Maturitas. 2017;103:8-15. doi:10.1016/j.maturitas.2017.06.020.

14. Castello A, Boldo E, Amiano P, Castaño-Vinyals G, Aragones N, Gómez-Acebo I, et al. Mediterranean dietary pattern is associated to low risk of aggressive prostate cancer: MCC-Spain study. J Urol. 2017 (In press).

15. Castano-Vinyals G, Aragones N, Perez-Gomez B, Martin V, Llorca J, Moreno V, et al. Population-based multicase-control study in common tumors in Spain (MCC-Spain): rationale and study design. Gaceta sanitaria/SESPAS. 2015;29:308-15. doi:10.1016/j.gaceta.2014.12.003.

16. Lope V, Fernandez de Larrea N, Perez-Gomez B, Martin V, Moreno V, Costas L, et al. Menstrual and reproductive factors and risk of gastric and colorectal cancer in Spain. PLoS ONE. 2016;11:e0164620. doi:10.1371/journal.pone.0164620.

17. Lauren $P$. The two histological main types of gastric carcinoma: diffuse and so-called intestinal-type carcinoma. An attempt at a histo-clinical classification. Acta Pathol Microbiol Scand. 1965;64:31-49.

18. Lauren PA, Nevalainen TJ. Epidemiology of intestinal and diffuse types of gastric carcinoma. A time-trend study in Finland with comparison between studies from high- and low-risk areas. Cancer (Phila). 1993;71:2926-33.

19. Garcia-Closas R, Garcia-Closas M, Kogevinas M, Malats N, Silverman D, Serra C, et al. Food, nutrient and heterocyclic amine intake and the risk of bladder cancer. Eur J Cancer. 2007;43:173140. doi:10.1016/j.ejca.2007.05.007.

20. Michel A, Waterboer T, Kist M, Pawlita M. Helicobacter pylori multiplex serology. Helicobacter. 2009;14:525-35. doi:10.1111/j.1523-5378.2009.00723.x.

21. Burt C. Factor analysis and canonical correlations. Br J Math Stat Psychol. 1948;1:95-106.

22. Castello A, Buijsse B, Martin M, Ruiz A, Casas AM, Baena-Canada JM, et al. Evaluating the applicability of data-driven dietary patterns to independent samples with a focus on measurement tools for pattern similarity. J Acad Nutr Diet. 2016;. doi:10.1016/j. jand.2016.05.008.

23. Castello A, Lope V, Vioque J, Santamarina C, Pedraz-Pingarron C, Abad S, et al. Reproducibility of data-driven dietary patterns in two groups of adult Spanish women from different studies. $\mathrm{Br}$ J Nutr. 2016;. doi:10.1017/S000711451600252X.

24. Levin ML. The occurrence of lung cancer in man. Acta Unio Int Contra Cancrum. 1953;9:531-41.

25. Hanley JA. A heuristic approach to the formulas for population attributable fraction. J Epidemiol Commun Health. 2001;55:508-14.

26. Bastos J, Lunet N, Peleteiro B, Lopes C, Barros H. Dietary patterns and gastric cancer in a Portuguese urban population. Int $\mathrm{J}$ Cancer. 2010;127:433-41. doi:10.1002/ijc.25013.

27. Campbell PT, Sloan M, Kreiger N. Dietary patterns and risk of incident gastric adenocarcinoma. Am J Epidemiol. 2008;167:295304. doi:10.1093/aje/kwm294.

28. Bahmanyar S, Ye W. Dietary patterns and risk of squamous-cell carcinoma and adenocarcinoma of the esophagus and adenocarcinoma of the gastric cardia: a population-based case-control study in Sweden. Nutr Cancer. 2006;54:171-8. doi:10.1207/ s15327914nc5402_3. 
29. Denova-Gutierrez E, Hernandez-Ramirez RU, Lopez-Carrillo L. Dietary patterns and gastric cancer risk in Mexico. Nutr Cancer. 2014;66:369-76. doi:10.1080/01635581.2014.884237.

30. De Stefani E, Deneo-Pellegrini H, Boffetta P, Ronco AL, Aune D, Acosta G, et al. Dietary patterns and risk of cancer: a factor analysis in Uruguay. Int J Cancer. 2009;124:1391-7. doi:10.1002/ ijc. 24035 .

31. Kim MK, Sasaki S, Sasazuki S, Tsugane S. Prospective study of three major dietary patterns and risk of gastric cancer in Japan. Int J Cancer. 2004;110:435-42. doi:10.1002/ijc.20132.

32. De Stefani E, Correa P, Boffetta P, Deneo-Pellegrini H, Ronco AL, Mendilaharsu M. Dietary patterns and risk of gastric cancer: a case-control study in Uruguay. Gastric Cancer. 2004;7:211-20. doi:10.1007/s10120-004-0295-2.

33. Masaki M, Sugimori H, Nakamura K, Tadera M. Dietary patterns and stomach cancer among middle-aged male workers in Tokyo. Asian Pac J Cancer Prev. 2003;4:61-6.

34. Palli D, Russo A, Decarli A. Dietary patterns, nutrient intake and gastric cancer in a high-risk area of Italy. Cancer Causes Control. 2001;12:163-72.

35. Bogovski P, Bogovski S. Animal species in which $N$-nitroso compounds induce cancer. Int J Cancer. 1981;27:471-4.

36. Trafialek J, Kolanowski W. Dietary exposure to meat-related carcinogenic substances: is there a way to estimate the risk? Int J Food Sci Nutr. 2014;65:774-80. doi:10.3109/09637486.2014.917146.
37. Reizenstein P. Iron, free radicals and cancer. Med Oncol Tumor Pharmacother. 1991;8:229-33.

38. Giugliano D, Ceriello A, Esposito K. The effects of diet on inflammation: emphasis on the metabolic syndrome. J Am Coll Cardiol. 2006;48:677-85. doi:10.1016/j.jacc.2006.03.052.

39. Fang YZ, Yang S, Wu G. Free radicals, antioxidants, and nutrition. Nutrition (Burbank). 2002;18:872-9.

40. Le Marchand L. Cancer preventive effects of flavonoids: a review. Biomed Pharmacother. 2002;56:296-301.

41. Iimuro M, Shibata H, Kawamori T, Matsumoto T, Arakawa T, Sugimura T, et al. Suppressive effects of garlic extract on Helicobacter pylori-induced gastritis in Mongolian gerbils. Cancer Lett. 2002;187:61-8.

42. Park JM, Kwon SH, Han YM, Hahm KB, Kim EH. Omega-3 polyunsaturated fatty acids as potential chemopreventive agent for gastrointestinal cancer. J Cancer Prev. 2013;18:201-8. doi:10.15430/ jcp.2013.18.3.201.

43. Calvert C, Cade J, Barrett JH, Woodhouse A. Using cross-check questions to address the problem of mis-reporting of specific food groups on Food Frequency Questionnaires. UKWCS Steering Group. United Kingdom Women's Cohort Study Steering Group. Eur J Clin Nutr. 1997;51:708-12.

44. Draugalis JR, Coons SJ, Plaza CM. Best practices for survey research reports: a synopsis for authors and reviewers. Am J Pharm Educ. 2008;72:11.

\section{Affiliations}

\section{Adela Castelló ${ }^{1,2,3}$ - Nerea Fernández de Larrea ${ }^{1,2} \cdot$ Vicente Martín $^{4} \cdot$ Verónica Dávila-Batista ${ }^{4}$. Elena Boldo ${ }^{1,2}$. Marcela Guevara ${ }^{2,5}$. Víctor Moreno ${ }^{2,6,7} \cdot$ Gemma Castaño-Vinyals $^{2,8,9,10} \cdot$ Inés Gómez-Acebo $^{11}$. Guillermo Fernández-Tardón ${ }^{12} \cdot$ Rosana Peiró $^{2,13} \cdot$ Rocío Olmedo-Requena $^{2,14,15} \cdot$ Rocio Capelo $^{16}$. Carmen Navarro $2,17,18$. Silvino Pacho-Valbuena ${ }^{19}$ • Beatriz Pérez-Gómez ${ }^{1,2}$ - Manolis Kogevinas ${ }^{2,8,9,10}$. Marina Pollán ${ }^{1,2} \cdot$ Nuria Aragonés ${ }^{1,2}$. On behalf of the MCC-Spain researchers}

1 Cancer Epidemiology Unit, National Centre for Epidemiology, Instituto de Salud Carlos III, Av/Monforte de Lemos, 5, 28029 Madrid, Spain

2 Consortium for Biomedical Research in Epidemiology \& Public Health (CIBERESP), Carlos III Institute of Health, Av/Monforte de Lemos, 5, 28029 Madrid, Spain

3 Faculty of Medicine, University of Alcalá, Alcalá de Henares, Madrid, Spain

4 The Research Group in Gene-Environment and Health Interactions, Vegazana Campus, University of León, León, Spain

5 Public Health Institute of Navarra, IdiSNA, Pamplona, Spain

6 Department of Clinical Sciences, Faculty of Medicine, University of Barcelona, Campus de Bellvitge, L'Hospitalet de Llobregat, Spain

7 Unit of Biomarkers and Susceptibility, Cancer Prevention and Control Program, Catalan Institute of Oncology (ICO), IDIBELL, Gran Via km 2.7, 08907 L'Hospitalet de Llobregat, Spain

8 ISGlobal, Centre for Research in Environmental Epidemiology (CREAL), Barcelona, Spain

9 Universitat Pompeu Fabra (UPF), Barcelona, Spain
10 IMIM (Hospital del Mar Medical Research Institute), Barcelona, Spain

11 Universidad de Cantabria-IDIVAL, Santander, Spain

12 IUOPA, University of Oviedo, Oviedo, Spain

13 Fundación para el Fomento de la Investigación Sanitaria y Biomédica de la Comunitat Valenciana FISABIO-Salud Pública, Valencia, Spain

14 Department of Preventive Medicine and Public Health, University of Granada, Granada, Spain

15 Instituto de Investigación Biosanitaria ibs.GRANADA. Complejo Hospitales Universitarios de Granada/Universidad de Granada, Granada, Spain

16 Centro de Investigación en Salud y Medio Ambiente (CYSMA), Universidad de Huelva, Huelva, Spain

17 Department of Epidemiology, Murcia Regional Health Council, IMIB-Arrixaca, Murcia, Spain

18 Department of Health and Social Sciences, Universidad de Murcia, Murcia, Spain

19 Servicio de Cirugía General, Complejo Asistencial Universitario de León, León, Spain 